\title{
Determinação da redução da resistência à tração em corpos de prova com Weld Line
}

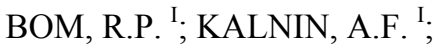 \\ ${ }^{\text {I }}$ Universidade do Estado de Santa Catarina - UDESC - Centro de Ciências Tecnológicas - CCT. \\ ${\text { Dept }{ }^{o} \text {. Eng }}^{\mathrm{a}}$. Mecânica, Mestrado em Ciência e Engenharia de Materiais, \\ Campus Universitário Avelino Marcante, B. Bom Retiro, CEP 89.201-100, Joinville, SC, Brasil \\ e-mail: dem2rpb@joinville.udesc.br, flaviokalnin@expresso.com.br
}

\section{RESUMO}

Neste trabalho objetivou-se determinar a redução da resistência à tração de corpos de prova com linhas de solda. Os corpos de prova ASTM foram moldados pelo processo de injeção com diferentes temperaturas de plastificação $\left(180^{\circ} \mathrm{C}\right.$ a $\left.280^{\circ} \mathrm{C}\right)$. O material utilizado foi o polímero termoplástico Polystyrol $158 \mathrm{~K}$ da Basf. Este estudo relaciona as linhas de solda em produtos moldados por injeção com a redução da resistência mecânica devido a fragilização na região da linha de solda.

Para o desenvolvimento deste trabalho foi projetado e desenvolvido um molde de injeção com uma cavidade em forma de corpo de prova com canal de ataque pelas extremidades. Desta forma no momento da junção dos fluxos obtêm-se uma linha de solda no centro do corpo de prova. Os parâmetros do processo de injeção foram determinados a partir de um aplicativo comercial. A temperatura do molde e o tempo de injeção permaneceram constantes. O tempo de solidificação do material foi determinado analiticamente, considerando-se o centro do corpo de prova como referência.

Foram injetados dez corpos de prova em cada uma das temperaturas. Foi observada de forma clara a existência de linhas de junção no centro da peça e as condições de processo utilizadas não permitiram a formação de linha de solda fria. Após a realização dos ensaios de tração, foi determinado que a tensão de ruptura decresce nas seguintes condições: com a diminuição da temperatura de injeção e do tempo de solidificação e com o aumento da trinca. A ruptura sempre ocorreu na linha de solda.

Palavras chave: Ensaio de tração, tempo de resfriamento, linha de solda, moldagem por injeção.

\section{Determination of reduction of the weld line strength in injection molded}

\section{ABSTRACT}

In this paper, the purpose is to determine the weld line strength in injection molded samples. The ASTM standard dogbone-shaped samples were molded over a range of melt temperatures $\left(180{ }^{\circ} \mathrm{C}\right.$ up to 280 ${ }^{\circ} \mathrm{C}$ ). The plastic used was a commercial grade of polystyrene (PS), BASF Polystyrol 158K. This study links weld lines in parts by injection molding with mechanical strength reduction due to embrittling effect in weldline region.

A single-cavity mold double-gated was used to generate ASTM D638 Type I tensile specimens. The double-gated and runner allow the parts to be molded with weld line. The injection process parameters were determined by imputing resin, machine and geometry information into a CAE software package. The mold temperature and fill time were maintaining the same. The frozen time was determined by analytic equation, which considers the center of the thickness as reference.

For each melt temperature 10 samples were tested. The weld line was clearly noted in the centre of the samples and the process condition set avoided the cold weld line. By the strength tests were possible to realize that lower the injection temperature lower the frozen time and higher the length of the crack. Then low injection temperature leads to low maximum strength. The fracture always occurs in the weld line.

Keywords: Strength test, cooling time, weld line, injection molding.

\section{INTRODUÇÃO}

No desenvolvimento de peças ou produtos em material plástico existem dois aspectos principais a serem abordados no que tange a formação de linhas de solda. O primeiro diz respeito à estética, linhas de 
solda não devem estar aparentes nas superfícies principais de um produto e, para que não ocorra isto elas são deslocadas para outras superfícies, ou então, a superfície de interesse pode ser texturizada com o objetivo de camuflar a marca da linha de junção ou de solda [1]. O segundo está correlacionado com o aspecto estrutural, ou seja, com a resistência na região de formação da linha de solda [2]. A formação da linha de solda está correlacionada com a separação e posterior união do fluxo dentro da cavidade do molde, os motivos podem ser vários, como por exemplo: múltiplos pontos de injeção, geometria complexa, variação na espessura que podem provocar o efeito de hesitação e refluxo, furos e rebaixos. A análise do ângulo de junção dos fluxos pode determinar se irá existir uma linha de junção ou solda [2, 3]. Vários pesquisadores têm empreendido esforços em estudos que abordam a redução da resistência devido à existência de linhas de soda e linhas de junção $[\underline{4}, \underline{5}, \underline{6}, \underline{7}, \underline{8}, \underline{9}$. Na linha solda existe a difusão molecular entre as duas superfícies, pois quanto maior for a temperatura e o tempo de permanência na temperatura acima da $T_{g}$ maior será a difusão na interface $[\underline{10}, \underline{11}, \underline{12}]$. Se houver formação de uma linha de solda fria, a adesão entre as interfaces se faz praticamente apenas pelo efeito da tensão superficial [13]. Assim, existe um tempo, uma temperatura e uma pressão ideal para se reconstituir uma interface de linha de solda. Se estas condições forem atingidas em um processo de moldagem por injeção, então a linha de solda estará otimizada. Neste trabalho será dado o enfoque na variação da tensão de ruptura em função da temperatura de injeção.

\section{MATERIAIS E MÉTODOS}

No desenvolvimento deste trabalho foi utilizado um polímero termoplástico de estrutura amorfa, Polystyrol $158 \mathrm{~K}$ da Basf. O material utilizado foi secado a $80^{\circ} \mathrm{C}$ durante duas horas antes de ser injetado. $\mathrm{Na}$ Figura 1 está representada uma fotografia da placa móvel do molde de injeção. A alimentação do corpo de provas foi feita pelas extremidades com um canal de ataque do tipo filme com espessura igual a $2 / 3$ da espessura do corpo de prova. A escolha deste tipo de canal de ataque foi feita para se obter uma frente de fluxo uniforme e os demais parâmetros de projeto foram consultadas as referências $[14, \underline{15}, \underline{16}, \underline{17}, \underline{18}]$. Os canais de ataque foram balanceados para que a linha de solda ficasse no meio do corpo de provas. A bucha de injeção que contém o canal principal pode ser rotacionada para se obter corpos de prova alimentados por um dos lados, como visto na figura, neste molde é possível obter amostras com e sem linha de solda.

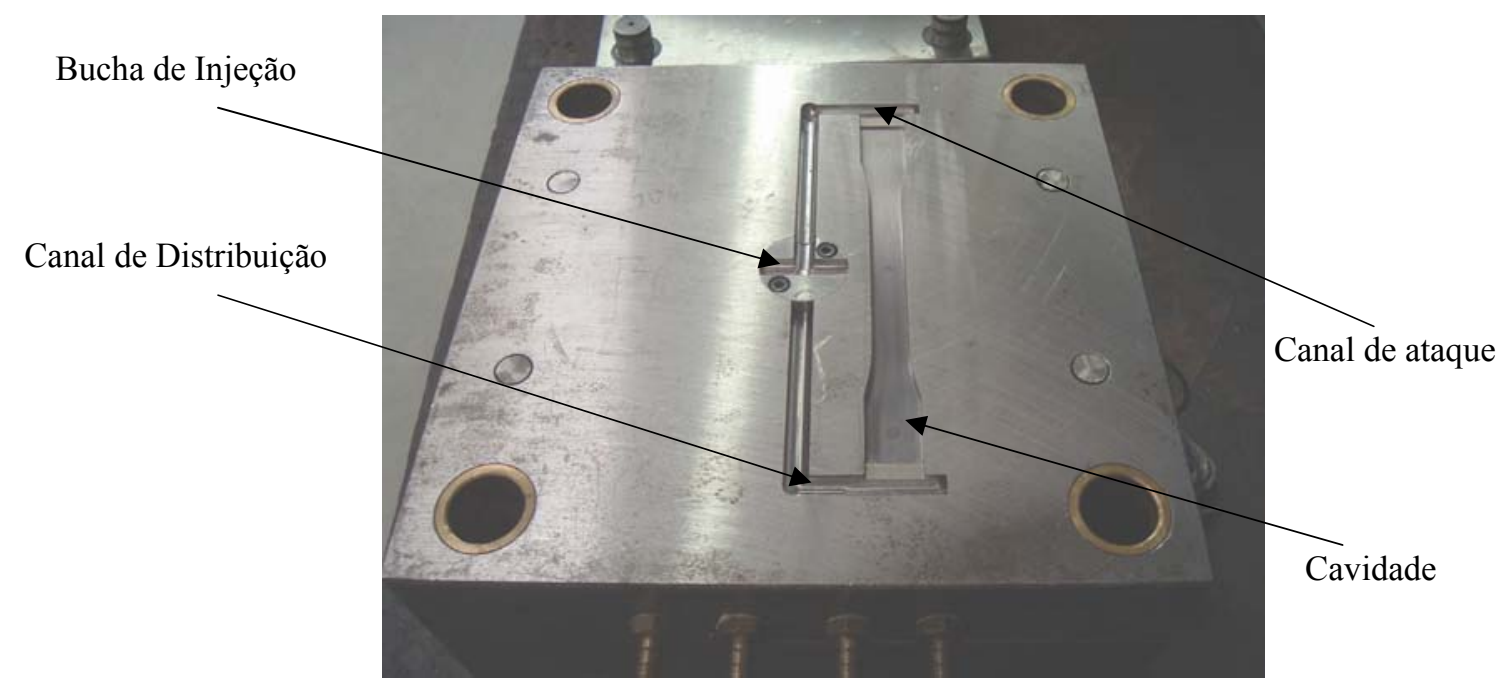

Figura 1: Fotografia da placa móvel do molde de injeção

Na Figura 2 está representada a forma final dos canais de distribuição e do corpo de prova formato ASTM D 638, tipo I, após o processo de injeção.

Utilizou-se no desenvolvimento experimental a injetora modelo 250 Plus, da marca Battenfeld, com as seguintes características: rosca de $22 \mathrm{~mm}$ de diâmetro e capacidade de injeção de $27 \mathrm{~g}$ de PE. 


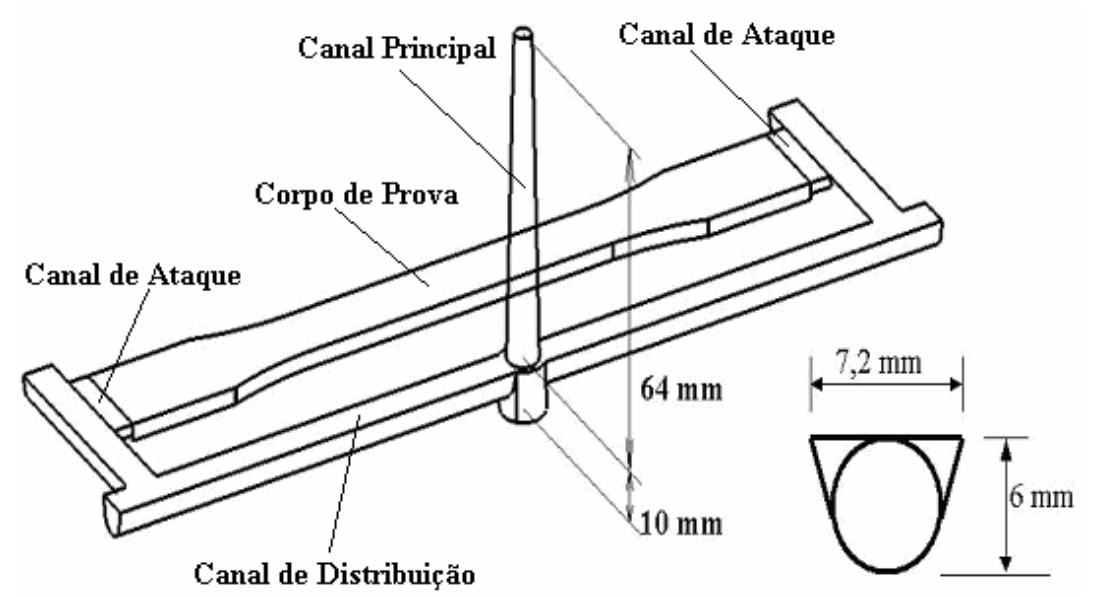

Figura 2: Forma das cavidades que formam o conjunto corpo de prova e canais de distribuição.

Foram utilizados os seguintes parâmetros de injeção determinados por simulação [3]], conforme indicado na Tabela 1.

Tabela 1. Parâmetros de processo de injeção determinados por simulação.

\begin{tabular}{lccccccc}
\hline Temperatura de injeção $\left({ }^{\mathrm{O}} \mathrm{C}\right)$ & 180 & 204 & 215 & 230 & 245 & 260 & 280 \\
\hline Temperatura do molde $\left({ }^{\mathrm{O}} \mathrm{C}\right)$ & 51 & 51 & 51 & 51 & 51 & 51 & 51 \\
Tempo de injeção (s) & 4,5 & 4,5 & 4,5 & 4,5 & 4,5 & 4,5 & 4,5 \\
Tempo de recalque (s) & 4,4 & 5,4 & 5,8 & 6,3 & 6,8 & 7,2 & 7,8 \\
Tempo de solidificação (s) & 19,2 & 21,7 & 22,7 & 23,9 & 25,1 & 26,1 & 27,3 \\
Pressão de injeção $(\mathrm{MPa})$ & 38,33 & 30,03 & 27,02 & 23,45 & 20,37 & 17,67 & 14,60 \\
Pressão de recalque (MPa) & 30,66 & 24,02 & 21,62 & 18,76 & 16,30 & 14,14 & 11,68 \\
\hline
\end{tabular}

Para os ensaios de tração utilizou-se uma máquina de tração, modelo DL 30.000 da empresa Emic, com célula de carga de $10.000 \mathrm{~N}$, extensômetro de $50 \mathrm{~mm}$ e velocidade de tração de $5 \mathrm{~mm} / \mathrm{min}$. Temperatura de ensaio de $26^{\circ} \mathrm{C}$ e umidade relativa do ar de $65 \%$.

\section{RESULTADOS E DISCUSSÃO}

A Equação 1 considera a transferência de calor por condução em uma placa de material plástico de $2 \mathbf{e}$ de espessura, a temperatura inicial desta placa é $\mathrm{T}_{\text {injeção }}$ no instante $\mathrm{t}=0$. A teoria prevê a aproximação de duas placas metálicas com temperatura constante [19] e por semelhança com o processo de injeção esta

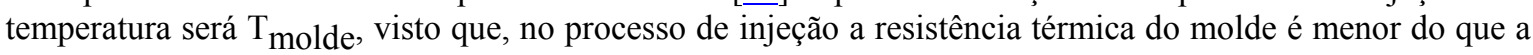
do material injetado $[14,20]$. A solução analítica da equação pode ser obtida para varias camadas da placa quando atribuídos os respectivos valores.

$$
\frac{\mathrm{T}_{\text {molde }}-\mathrm{T}(\mathrm{y}, \mathrm{t})}{\mathrm{T}_{\text {molde }}-\mathrm{T}_{\text {injeção }}}=2 \sum_{\mathrm{n}=\mathrm{o}}^{\infty} \frac{(-1)^{\mathrm{n}}}{\left(\mathrm{n}+\frac{1}{2}\right) \pi} \exp \left[-\left(\mathrm{n}+\frac{1}{2}\right)^{2} \pi^{2} \frac{\alpha \mathrm{t}}{\mathrm{e}^{2}}\right] \cos \left[\left(\mathrm{n}+\frac{1}{2}\right) \frac{\pi \mathrm{y}}{\mathrm{e}}\right]
$$

A temperatura do molde ( $\mathrm{T}_{\text {molde }}$ ) foi mantida constante em $51{ }^{\circ} \mathrm{C}$, as temperaturas de injeção $\left(\mathrm{T}_{\text {injeção }}\right)$ variaram de 180 a $280^{\circ} \mathrm{C}$. A temperatura na posição y no tempo $\mathrm{t},\left(\mathrm{T}_{(\mathrm{y}, \mathrm{t})}\right)$ é a variável a ser determinada, o número de termos da série utilizado foi de $\mathrm{n}=100$. O tempo $(\mathrm{t})$ variou de 0 a $40 \mathrm{~s}$, a espessura do corpo de prova foi de $3,35 \mathrm{~mm}$, portanto e=1,675 $\mathrm{mm}$. Foram calculados os tempos de solidificação do centro da placa que é o último lugar a solidificar (convém ressaltar que o tempo de solidificação deste trabalho é definido como aquele necessário para que o polímero resfrie até a temperatura de transição vítrea, $\mathrm{T}_{\mathrm{g}}$ ), para a solução da equação é considerado $\mathrm{y}=0$. A difusividade térmica do material $(\alpha)$ é calculada a partir da Equação 2, o valor de $\alpha$ utilizado foi de $\alpha=0,0744 \mathrm{~mm} / \mathrm{s}^{2}$. A condutividade térmica (K) do PS utilizado foi 
de $\mathrm{K}=0,140 \mathrm{~W} / \mathrm{m}^{\mathrm{O}} \mathrm{C}$, a massa específica $(\rho)$ foi considerada constante para o material plastificado, $\rho=0,955 \mathrm{~g} / \mathrm{cm}^{3}$ e o calor específico do material como sendo $\mathrm{C}_{\mathrm{p}}=1.970 \mathrm{~J} / \mathrm{kg}^{\mathrm{O}} \mathrm{C}$.

$$
\alpha=\frac{k}{\rho C p}
$$

No gráfico ilustrado, na Figura 3, estão representadas as curvas obtidas pela solução analítica da Equação 1. No início de cada curva para $\mathrm{t}=0$ tem-se a temperatura de injeção $\mathrm{T}_{\text {injeção, a temperatura do }}$ centro da placa vai diminuindo com o tempo. Traçando uma reta representando a temperatura de transição vítrea do material $\mathrm{Tg}=100^{\circ} \mathrm{C}$, a interseção desta reta da $\mathrm{Tg}$ com as demais curvas permite obter o tempo de solidificação do corpo de prova. Na Tabela 2 estão indicados os tempos assim determinados. A linha logo abaixo indica a temperatura de desmoldagem ou extração. Mais abaixo, está representado uma linha indicando a temperatura do molde de $51^{\circ} \mathrm{C}$, como pode ser observado pelo resultado da simulação, nenhum dos casos atingiu a temperatura do molde em 40 s.

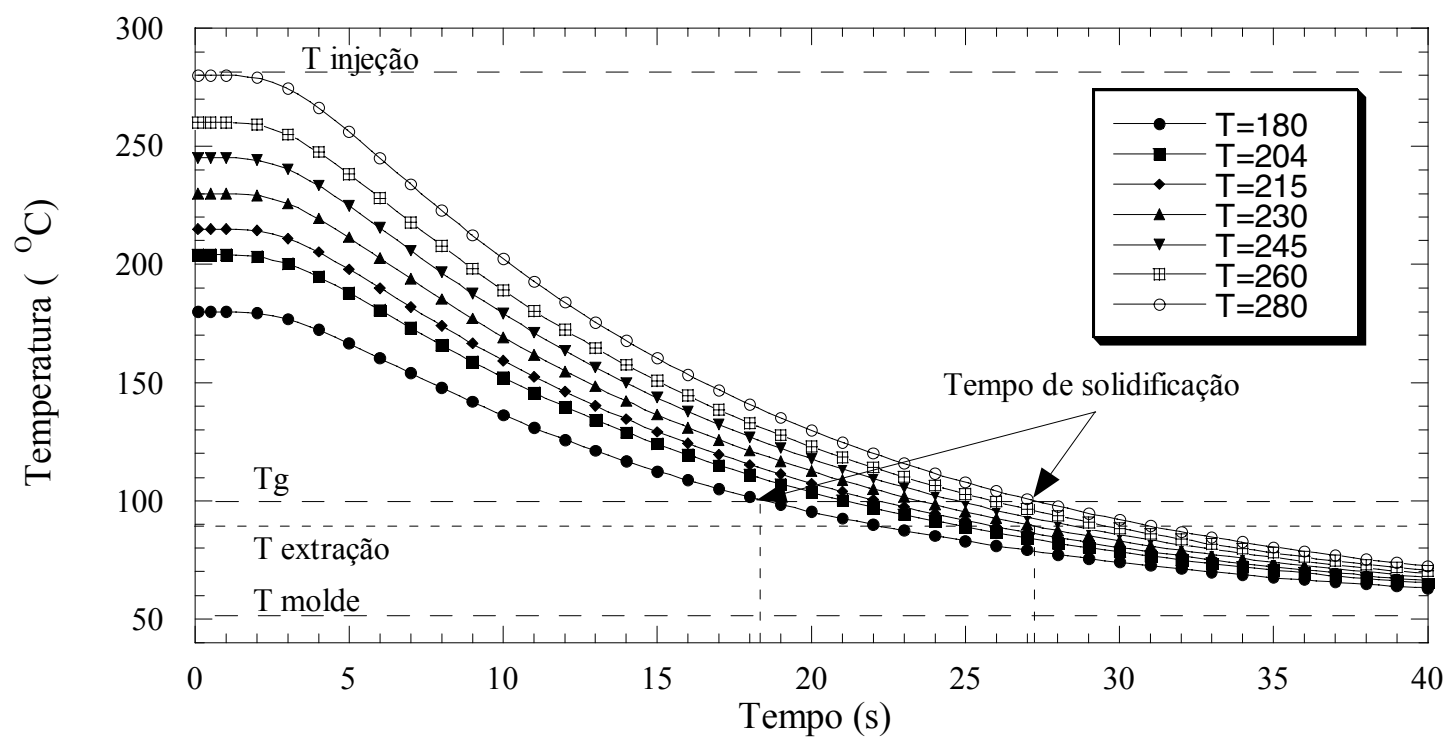

Figura 3: Variação da temperatura ao longo do tempo para $y=0$ e para diferentes temperaturas de injeção.

Os valores médios das tensões de ruptura obtidas a partir dos ensaios de tração dos corpos de prova, com e sem linhas de solda, e seus respectivos desvios padrão, estão indicados na Tabela 2. A área total do corpo de prova sem linha de solda $\left(\mathrm{A}_{\mathrm{O}}\right)$ é igual a $43,55 \mathrm{~mm}^{2}$. A tensão $\sigma_{\mathrm{O}}$ é a tensão máxima de ruptura obtida também com corpos de prova sem linhas de solda. A tensão $\sigma_{\omega}$ é a tensão de ruptura determinada para os corpos de prova com linhas de solda. Deve-se salientar que devido à característica frágil do material, a tensão máxima e a tensão de ruptura são as mesmas. A forma em $\mathrm{V}$ da linha de solda/entalhe pode ser observada na Figura 4. A linha de solda está presente em todo o perímetro da seção central do corpo de prova. A trinca que está sendo abordada neste trabalho compreende não apenas o entalhe em $\mathrm{V}$ observado, mas sim toda a região onde a interpenetração molecular não é suficiente para refazer a interface de junção dos dois fluxos, no caso representado por "a" na Figura 4. A profundidade do entalhe V gerado durante a fase de preenchimento mostrada na Figura 4 é de aproximadamente $\mathrm{h}=10 \mu \mathrm{m}$. O entalhe $\mathrm{V}$ é uma característica do processo de injeção quando dois fluxos se encontram, nesta região por efeitos de aprisionamento de ar no molde, falta de pressão e temperatura, não permitem a reconstituição da interface, a interpenetração molecular fica prejudicada também abaixo do vértice do entalhe em V. Por isto, a trinca "a" é considerada da superfície do corpo de provas até uma certa profundidade, determinada como mostrado pelas Equações 3 e 4.

A solução da Equação 3 permite determinar a área da trinca $\left(\mathrm{A}_{\text {Trinca }}\right)$ [4] , considerando a seção transversal do corpo de provas um retângulo perfeito e que a trinca como sendo igual em todo o perímetro, então, a solução das Equações 3 e 4 permitiu determinar o tamanho da trinca, "a", para cada temperatura de injeção. 


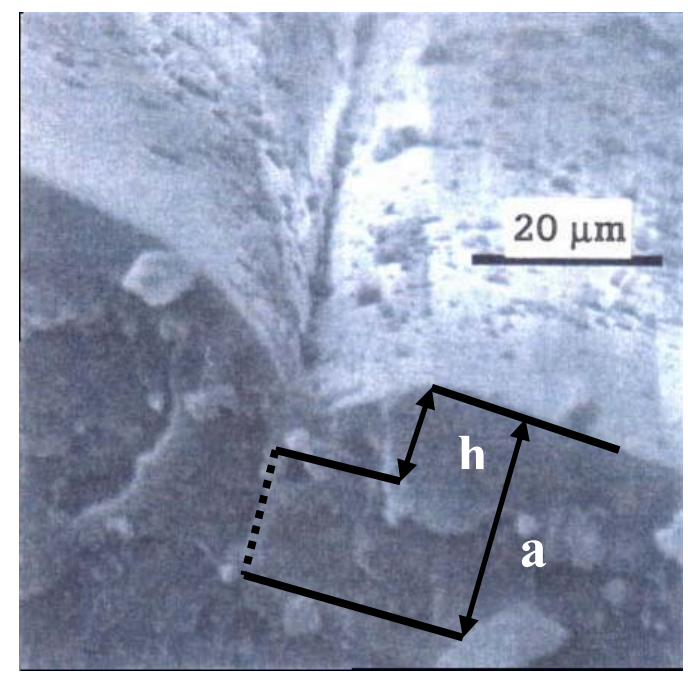

Figura 4: Linha de solda com entalhe com formato V e profundidade h, trinca "a", Polystyrol $158 \mathrm{~K}[\underline{1}, \underline{2}]$

Para a determinação da tensão máxima $\sigma_{\mathrm{O}}$ para o corpo de prova sem weld line foi adotada a temperatura média de processamento do $\mathrm{PS}$ de $230^{\circ} \mathrm{C}$. Sabe-se que a tensão pode ter um pouco de variação dentro da faixa recomendada de processamento. Provavelmente devido à estrutura amorfa o empacotamento molecular não deve alterar muito, entretanto, este último dependente de vários fatores como, por exemplo, temperatura de injeção, pressão de injeção.

$$
\sigma_{\omega}=\sigma_{0}\left(\frac{A_{O}-A_{\text {trinca }}}{A_{0}}\right)
$$

Os resultados das Equações 3 e 4 estão indicados na Tabela 2, onde "a" é o tamanho da trinca.

$$
4 \cdot \mathrm{a}^{2}-32,70 \cdot \mathrm{a}+\mathrm{A}_{\text {Trinca }}=0
$$
6.

O percentual de redução da tensão (\%), indicado na Tabela 2, são calculados por meio da Equação

Tabela 2: Valores da temperatura de injeção, $\sigma_{\omega}, \sigma_{0}$, desvio padrão, $A_{\text {Trinca }}, A_{0}$, percentual de redução da tensão (\%), trinca e tempo para que $\mathrm{y}=0$ atinja a $\mathrm{T}_{\mathrm{g}}$.

\begin{tabular}{ccccccc}
\hline $\begin{array}{c}\text { Temperatura } \\
\text { de injeção } \\
\left({ }^{\circ} \mathrm{C}\right)\end{array}$ & $\begin{array}{c}\sigma_{\omega} \\
(\mathrm{MPa})\end{array}$ & $\begin{array}{c}\text { Desvio } \\
\text { Padrão }\end{array}$ & $\begin{array}{c}\mathrm{A}_{\text {Trinca }} \\
\left(\mathrm{mm}^{2}\right)\end{array}$ & $\begin{array}{c}\text { Redução da } \\
\text { Tensão } \\
(\%)\end{array}$ & $\begin{array}{c}\text { Trinca “a” } \\
(\mathrm{mm})\end{array}$ & $\begin{array}{c}\text { Tempo } \\
(\mathrm{s})\end{array}$ \\
\hline 180,0 & 22,56 & 2,37 & 21,016 & 48,26 & 0,703 & 18,45 \\
204,0 & 26,74 & 0,74 & 16,841 & 38,67 & 0,552 & 21,00 \\
215,0 & 28,82 & 1,32 & 14,763 & 33,90 & 0,479 & 22,18 \\
230,0 & 31,77 & 1,06 & 11,816 & 27,13 & 0,378 & 23,50 \\
245,0 & 34,57 & 1,25 & 9,0196 & 20,71 & 0,285 & 24,67 \\
260,0 & 37,04 & 1,74 & 6,5525 & 15,05 & 0,205 & 25,80 \\
280,0 & 38,65 & 1,33 & 4,9443 & 11,35 & 0,154 & 27,24 \\
$\begin{array}{c}\text { Sem linha } \\
\text { de solda }\end{array}$ & $\begin{array}{c}\sigma_{0}= \\
43,60\end{array}$ & 1,04 & $\begin{array}{c}\mathrm{A}_{0}= \\
43,550\end{array}$ & 0.00 & 0,000 & \\
\hline
\end{tabular}


Comparando os resultados da Tabela 1 com os da Tabela 2, para o tempo de solidificação determinado para diferentes temperaturas. Sendo o primeiro obtido a partir de simulação com o aplicativo comercial e o segundo determinado analiticamente pela Equação 1, pode-se observar que existe coerência entre os resultados calculados a partir dos parâmetros característicos do material e utilizados na Equação 1 .

A Figura 5 representa a variação da tensão de ruptura com os respectivos desvios padrão e o percentual de redução da tensão de ruptura em função da temperatura de injeção. Como pode ser observado, a tensão aumenta com o aumento da temperatura de injeção, ou seja, a linha de soda é melhorada com o aumento da temperatura de injeção. Entretanto, na temperatura máxima de processamento recomendada pelo fabricante não se atinge o máximo como obtido com o corpo de prova sem linha de solda. O percentual mínimo de perda foi de $11,35 \%$ e o máximo de perda ficou em $48,26 \%$ para a temperatura de $180^{\circ} \mathrm{C}$.

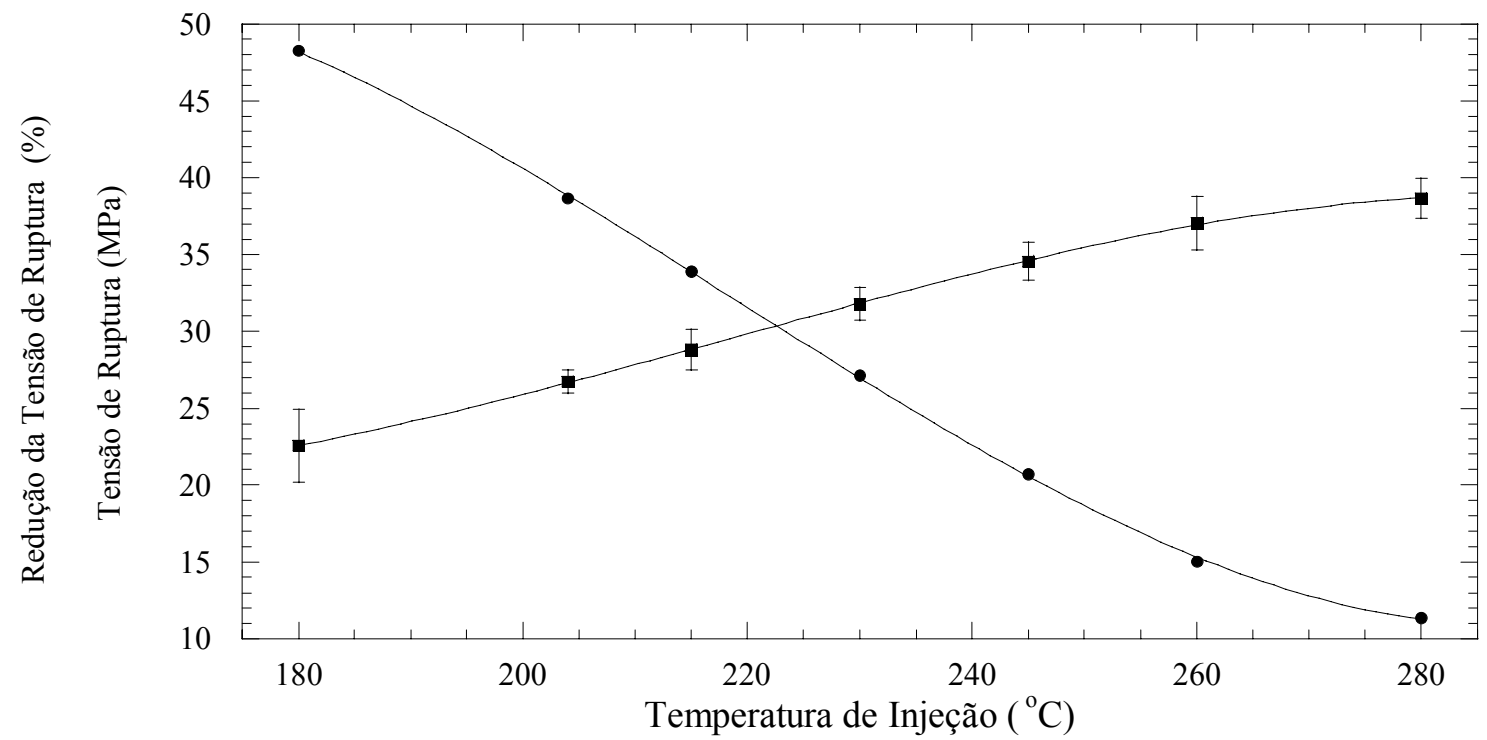

Figura 5. Variação da $\mathbf{\square}$ ) tensão de ruptura e • ) percentual de redução da tensão em função da temperatura de injeção.

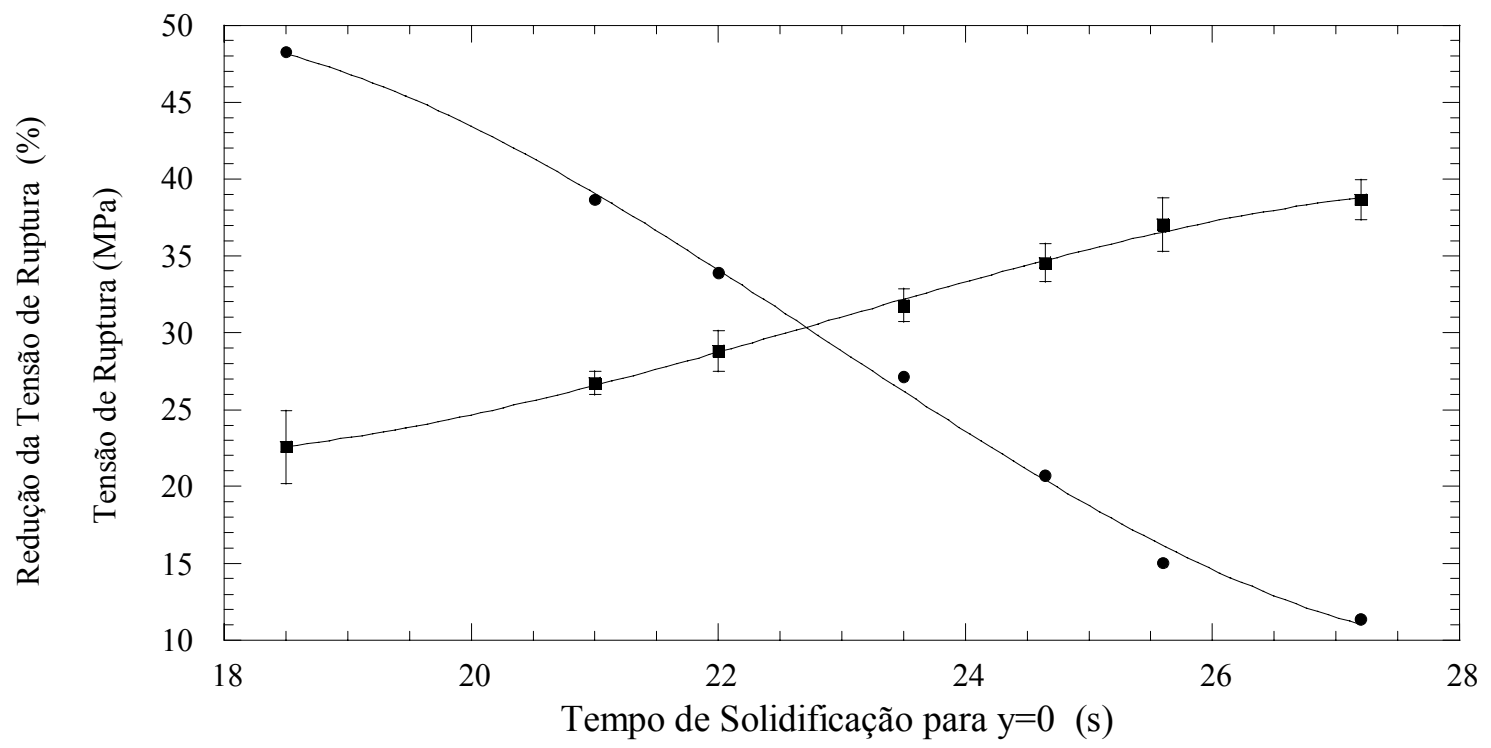

Figura 6. Variação da $\boldsymbol{\square}$ ) tensão de ruptura e • ) percentual de redução da tensão em função do tempo de solidificação. 
Na Figura 6, está representada a variação da tensão de ruptura em função do tempo de solidificação. Como pode ser observado, com maiores tempos de solidificação maior é a tensão de ruptura do PS, a dependência não é linear, sobre os pontos foi interpolado um polinômio de terceiro grau.

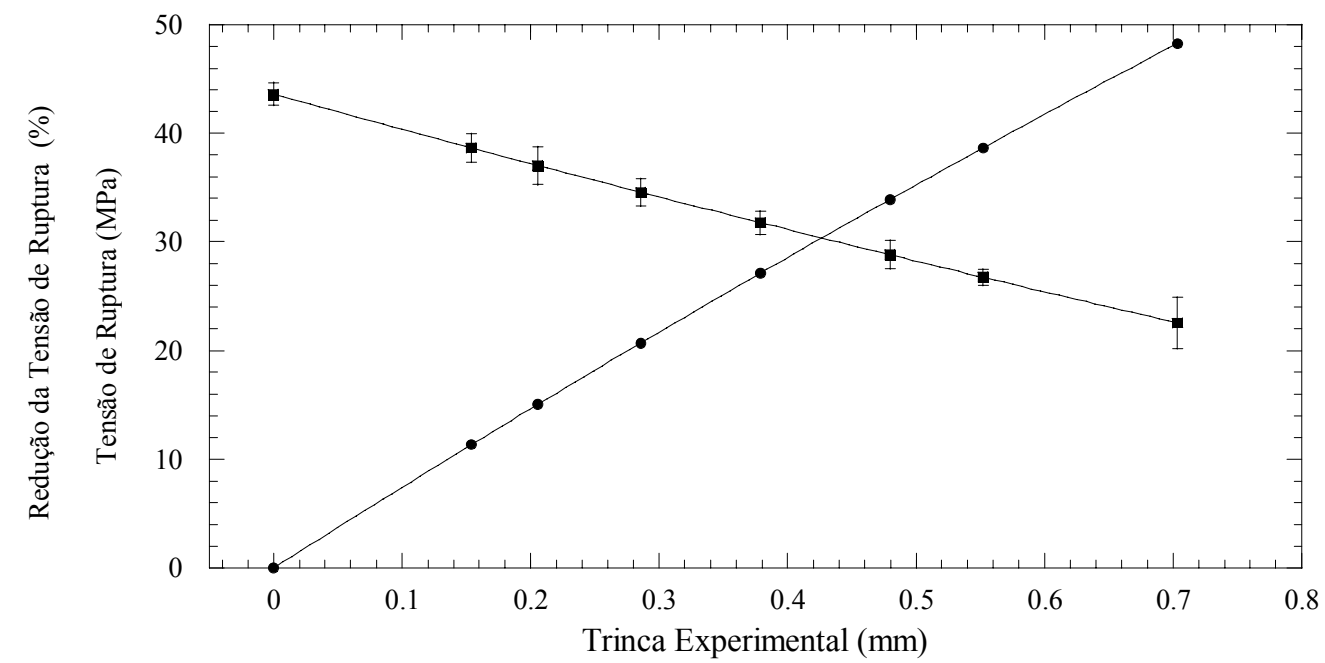

Figura 7: Variação da $\mathbf{\square}$ ) tensão de ruptura e • ) percentual de redução da tensão em função da dimensão da trinca.

Na Figura 7 estão representadas as curvas da variação da tensão de ruptura e seus respectivos desvios padrão e o percentual de redução da tensão em função da trinca experimental. Sobre os pontos foram interpoladas as seguintes equações: Inserindo a Equação 4 na Equação 3, resulta na Equação 5, portanto, a variação da tensão de ruptura em função da trinca pode ser representada por um polinômio de grau 2.

$$
\sigma_{\omega}=\sigma_{0}\left(\frac{A_{0}-4 \cdot a^{2}+32,70 . a}{A_{0}}\right)
$$

Re-escrevendo a ordem dos termos da Equação 5, chega-se a Equação 6, que representa a variação percentual de perda de tensão de ruptura em relação ao tamanho da trinca. A perda de tensão é dada pela diferença entre as tensões máximas dos corpos de prova com/sem weld line divididos pela tensão do corpo de prova sem weld line, multiplicados respectivamente por 100, para se obter o resultado em percentual.

$$
\left|\frac{\sigma_{\omega}-\sigma_{0}}{\sigma_{0}}\right| \times 100(\%)=\frac{\left(-4 . a^{2}+32,70 . a\right)}{A_{0}} \times 100
$$

\section{4}

\section{CONCLUSÕES}

A conclusão principal deste trabalho é que a temperatura na região de formação da linha de solda é o principal parâmetro que influencia na formação e na melhoria da reconstituição da interface. Neste estudo esta temperatura foi considerada igual à temperatura de injeção. A variação da tensão de ruptura em relação à variação da temperatura não apresentou um comportamento linear. O tempo de solidificação depende da temperatura de injeção e da espessura do corpo de provas, sendo maior quanto maior for a temperatura de injeção. A variação da tensão de ruptura foi descrita a partir de uma equação que tem como variável o tamanho da trinca. Pôde-se verificar que aumentando o tamanho da trinca, diminui-se a tensão de ruptura do corpo de provas. Foi possível quantificar o percentual de redução da tensão de ruptura em função do tamanho da trinca. A ruptura do corpo de prova sempre ocorreu na linha de solda. Os resultados obtidos com as soluções analíticas da equação de solidificação de placas foram coerentes com os resultados obtidos com o aplicativo comercial. 


\section{BIBLIOGRAFIA}

[1] PINTO, M.A.G., BOM, R.P., Aspectos estéticos e estruturais de termoplásticos injetados: linhas de junção e de solda fria, Plástico Industrial, Ed. Aranda, pp. 146, 153, setembro de 2001.

[2] PINTO, M.A.G., "Analise dos resultados de simulação de injeção em aplicativo comercial, entre modelos matemáticos baseados em casca média e casca externa", Dissertação M.Sc., Universidade do Estado de Santa Catarina, , pp.111, Joinville, 2001.

[3] C-MOLD, C-mold Design Guide, Product Number D-701, Version 969.0498, Copyright@ 1994-1998 Advenced CAE Tecnology, Inc.

[4] KIM, S.G., SUH, N.P., "Performance prediction of weldline structure in amorphous polymers", Polymer Engineering Science, v. 26, n. 17, 1986.

[5] CHANG, T.; FAISON, E., Optimization of weld line in injection molding via an experimental design approach, Iowa State University, 1998

[6] O'BRIEN-SOUCY, A.K., BARRY, C. M. F., STACER R. G., The development of weld line strength in injection molded PMMA, University of Massachusetts, 1999.

[7] SHAOYUN, G., Ait-KADI, A., BOUSMINA, M. A., A modified model predictions and experimentalresults of weld line strength in injection molded PS/PMMA blends, Elsevier, 2004

[8] FELLAHI, S., Weld lines in injection-molded parts, Advanced Polymer Technology, 1995.

[9] MALGUARNERA, S.C., MANISALI, A., The structure and properties of weld line in injection molded thermoplastics, SPE ANTEC Tech. Papers, 1980.

[10] PECORINI, T.J., "A fracture mechanics approach to weld line frature in a amorphous cellulose acetate propionate", Polymer Engineering and Science v. 37, n. 2, 1997.

[11] WOOL, R.P., Polymer Interfaces: Structure and Strength, 1a. ed., New York, Elsevier Applied Science, 1989.

[12] DE GENNES, P. G., Scaling concepts in polymers physics, 1a. ed., New York, Cornell University Press, 1979.

[13] WOOL, R.P., O' CONNOR, K.M., “A Theory of Crack Healing in Polymers”, Jornal Applied Physics v. 52, n. 10, pp. 5951, 1981.

[14] BOM, R.P., "Efeito da Pressão e do Tempo de Recalque no Empacotamento de Peças Injetadas", Monografia, Ciência e Engenharia de Materiais no Centro de Ciências Tecnológicas, Universidade do Estado de Santa Catarina, UDESC, pp. 22-27, junho 2002.

[15] SORS, L., BARDDÓCZ, L., RADNÓTI, I., Plásticos Moldes \& Matrizes. São Paulo: Ed. Hemus, pp. $144-151$.

[16] GlanVILl A.B., DeNTON E.N., Princípios Básicos e Projetos. Moldes de Injeção, São Paulo: Edgard Blücher Ltda, 1980.

[17] DA CRUZ, S., Moldes de Injeção. São Paulo: Ed. Hemus, pp. 75-79.

[18] PROVENZA, F., Moldes para Plásticos, Ed. F. Provenza, pp.5.105-5.122, 1991.

[19] AGASSANT, J.F., AVENAS, P., SERGENT, J.P., VICENT, M., La Mise em Forme dês Matières Plastiques, 3 ed., Paris: Ed. Tec \& Doc Lavoisier, pp. 137-147, 1996.

[20] DA CRUZ, A.C., "Determinação do Tempo de Solidificação de Peças Termoplásticas Moldadas pelo Processo de Injeção", Dissertação de M. Sc., Ciência e Engenharia de Materiais, Centro de Ciências Tecnológicas, Universidade do Estado de Santa Catarina, UDESC, pp. 21 e 69, julho 2005. 